\title{
Network topology near criticality in adaptive epidemics
}

\author{
Leonhard Horstmeyer, ${ }^{1,2}$ Christian Kuehn, ${ }^{3,2}$ and Stefan Thurner ${ }^{1,2,4,5}$ \\ ${ }^{1}$ Section for the Science of Complex Systems, CeMSIIS, Medical University of Vienna, Spitalgasse 23, A-1090, Vienna, Austria \\ ${ }^{2}$ Complexity Science Hub Vienna, Josefstädterstrasse 39, A-1090 Vienna, Austria \\ ${ }^{3}$ Faculty of Mathematics, Technical University of Munich, Boltzmannstr. 3, 85748 Garching München, Germany \\ ${ }^{4}$ Santa Fe Institute, 1399 Hyde Park Road, Santa Fe, New Mexico 87501, USA \\ ${ }^{5}$ IIASA, Schlossplatz 1, 2361 Laxenburg, Austria
}

(Received 28 May 2018; published 29 October 2018)

\begin{abstract}
We study structural changes of adaptive networks in the coevolutionary susceptible-infected-susceptible (SIS) network model along its phase transition. We clarify to what extent these changes can be used as early-warning signs for the transition at the critical infection rate $\lambda_{c}$ at which the network collapses and the system disintegrates. We analyze the interplay between topology and node-state dynamics near criticality. Several network measures exhibit clear maxima or minima close to the critical threshold and could potentially serve as early-warning signs. These measures include the SI link density, triplet densities, clustering, assortativity, and the eigenvalue gap. For the SI link density and triplet densities the maximum is found to originate from the coexistence of two power laws. Other network quantities, such as the degree, the branching ratio, or the harmonic mean distance, show scaling with a singularity at $\lambda=0$ (not at $\lambda_{c}$ ), which means that they are incapable of detecting the transition.
\end{abstract}

DOI: 10.1103/PhysRevE.98.042313

\section{INTRODUCTION}

In recent years there has been an increasing focus on adaptive (or coevolving) networks [1-3]. The essence of adaptive networks is that node-state dynamics influences the network topology - and topology influences the node dynamics. Several adaptive network models have been phrased in the context of epidemics [4,5], game theory [6-8], sociodynamics [9], self-organized criticality [10,11], financial markets [12], and evolution [13], just to name a few. These models are understood, either by simulations or by appropriate approximations, such as mean-field approximations and moment closure $[9,14-16]$.

Adaptive networks show bifurcations or phase transitions, which means that they exist in at least two phases: In one phase the network is well connected, and in the other it has a drastically reduced density of links of a certain type $[1,17]$ or in general $[9,13]$ ("collapsed" phase). The corresponding critical parameters separate the phases and can be computed explicitly for several models. It is known that for bifurcationinduced critical transitions, in the vicinity of these critical parameters (tipping points), so-called early-warning signs (precursor signals) exist that are linked to the phenomenon of critical slowing down, see, e.g., Ref. [18]. For stochastic systems, slowing down can often be quantified by the autocorrelation and variance of the process. In the context of adaptive networks critical slowing down is observed in terms of node properties $[19,20]$.

A classic model for adaptive networks is the coevolving SIS model, where the term coevolving means that links and states - S (susceptible) and I (infected) - do not evolve independently. In the static SIS network model, where the network topology does not change over time, nodes are in the $\mathrm{S}$ or I state. Each infected node recovers from infection at a rate $r$. An infected node can transmit the disease to connected susceptible nodes at a rate $\lambda$. In Ref. [4] rewiring was introduced, where susceptible nodes may rewire a link from an infected node to a susceptible node at a rate $w$. This adaptive SIS model shows a different phase diagram: Besides the disease-free phase (all nodes in state $S$ ) and the endemic phase (a nonzero fraction of I nodes persists), which also exist in the classical model [21], it includes a bistable phase and an oscillatory phase $[4,5,22]$. In the following, we focus on the phase transition from the endemic or bistable phase to the disease-free state. The transition happens at a critical infection rate, the so-called the persistence threshold, $\lambda_{c}$.

The investigation of early-warning signs for this transition has three potential benefits. First, it can inform decision making for appropriate measures to control the disease: One can assess whether little effort is sufficient, say, in terms of hygiene and medical treatment, or whether major structural efforts are required, such as quarantines. For example, an early warning signal could tell decision makers how far an ongoing endemic is from the critical point where it cannot sustain itself. This information could be used to estimate the additional vaccination efforts necessary, to drive the effective infection rate below the critical persistance threshold, or to end the endemic. The larger the distance of the system to the critical infection rate, the stronger the measures need to be. Second, if the contagion process is not a malicious disease, but information or some benign contagion, then early-warning signs can help us to increase efforts to sustain its spreading. Last, some of the lessons learned from this study of precursors might be general, so that they can be applied to adaptive networks in general and thus can be transferred to other contexts.

The adaptive SIS model can be described with "macroscopic equations", where the stochastic node and link update 
dynamics is reduced to a system of ordinary differential equations that governs the fraction of infected nodes and the densities of the various link types in the population. The equations are derived in the so-called homogeneous pair approximation (PA). It is possible to estimate the critical infection rate at the persistence threshold. We denote the fraction of infected nodes by $\rho=[\mathrm{I}] / N$, where $N$ is the number of nodes. The per-node density of SS links, SI links, and II links are denoted by $\rho_{\mathrm{SS}}=[\mathrm{SS}] / N, \rho_{\mathrm{SI}}=[\mathrm{SI}] / N$, and $\rho_{\mathrm{II}}=[\mathrm{II}] / N$, respectively. We also consider the densities of the motifs, $\rho_{\mathrm{SSI}}=[\mathrm{SSI}] / N$ and $\rho_{\mathrm{ISI}}=[\mathrm{ISI}] / N$, which denote the respective triplet density per node. These densities are random variables, however, we denote their expectation values with the same variables. The evolution equations for the expectation values (up to second order) are given by [4]

$$
\begin{aligned}
\frac{d \rho}{d t} & =\lambda \rho_{\mathrm{SI}}-r \rho, \\
\frac{d \rho_{\mathrm{II}}}{d t} & =\lambda \rho_{\mathrm{SI}}+\lambda \rho_{\mathrm{ISI}}-2 r \rho_{\mathrm{II}}, \\
\frac{d \rho_{\mathrm{SS}}}{d t} & =(r+w) \rho_{\mathrm{SI}}-\lambda \rho_{\mathrm{SSI}} .
\end{aligned}
$$

Let $\langle k\rangle$ denote the average degree and note that since the total link density,

$$
\rho_{\mathrm{SS}}+\rho_{\mathrm{SI}}+\rho_{\mathrm{II}}=\frac{\langle k\rangle}{2},
$$

is conserved in the rewiring process, the seemingly missing $\rho_{\mathrm{SI}}$ equation can be eliminated. Equations (1b) and (1c) are not closed because they depend on triplet densities. To close them, one can use, e.g., the homogeneous pair approximation ${ }^{1}$ that neglects correlations between links,

$$
\rho_{\mathrm{SSI}} \approx 2 \frac{\rho_{\mathrm{SI}} \rho_{\mathrm{SS}}}{1-\rho}, \quad \rho_{\mathrm{ISI}} \approx \frac{\rho_{\mathrm{SI}} \rho_{\mathrm{SI}}}{1-\rho} .
$$

One can now solve for the stationary solution of the PA. The disease-free state is always a steady state but loses stability at the so-called invasion threshold, for which the PA yields $\lambda^{\text {invasion }}=(r+w) /\langle k\rangle$. In the PA the endemic state starts to be stable at the persistence threshold,

$$
\lambda_{c}=\frac{2 r}{\mu^{2}}\left(\sqrt{1+\frac{w \mu^{2}}{r}}-1\right),
$$

where we define $\mu:=\langle k\rangle-1$ as the approximate average excess degree. For $r \ll w \mu^{2}$, we have $\lambda_{c} \approx 2 \sqrt{w r} / \mu$. Figure 1(a) shows two time series of the prevalence $\rho$ in a simulation of the adaptive SIS model in the bistable regime. For the specific initial conditions shown, the dynamics either enters the stationary endemic state or the disease-free state. The smaller the initial disease prevalence, the higher the probability of ending up in the disease-free state. If the system is in the endemic state, then it explores its phase space stochastically. In Fig. 1(b) we show the average prevalence as a function of the infection rate $\lambda$. It asymptotically approaches

\footnotetext{
${ }^{1}$ The quality of this approximation can be checked in simulations, see Appendix A.
}
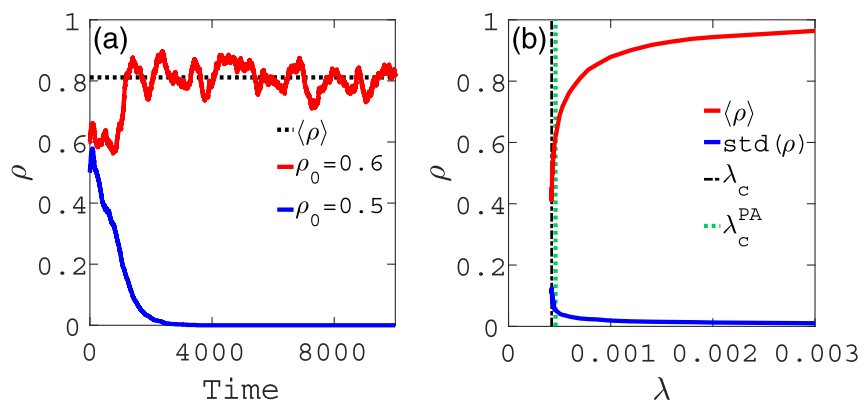

FIG. 1. (a) Disease prevalence in the adaptive SIS model. Two time series are shown with an initial prevalence of $60 \%$ (red) and $50 \%$ (blue). The network is initialized as an Erdös Rényi graph of size $N=400$ with an average degree of $\langle k\rangle=20$ and the remaining parameters are $r=0.002, \lambda=0.001$, and $w=0.01$. The dashed line indicates the stationary value in the endemic state. (b) Stationary endemic prevalence as a function of infection rates (red) and its standard deviation (blue) are shown for the same parameters. The numerical (green dotted) and pair approximation (black dashed) values of the persistence threshold are also indicated. Their values for these parameters are $\lambda_{c}=4.2 \times 10^{-4}\left( \pm 0.2 \times 10^{-4}\right)$ and $\lambda_{c}^{P A}=$ $4.6 \times 10^{-4}$ [cf. Equation (4)], respectively.

1 for large $\lambda$. Close to $\lambda_{c}$, the prevalence decreases sharply and eventually the endemic state ceases to exist. The standard deviation of $\rho$ increases as the infection rate approaches $\lambda_{c}$ from above [20]. This reflects that around the critical point, fluctuations become larger-the chance of an extinction event increases. Setting the right-hand side of (1) to zero and solving for $\rho$ yields the equilibrium curve for the prevalence in the PA and shows the leading order $\rho \propto\left(\lambda-\lambda_{c}\right)^{\frac{1}{2}}$ behavior,

$$
\rho=1-\frac{\lambda \mu}{2(w-\lambda)}+\frac{\sqrt{\lambda^{2} \mu^{2}-4 r(w-\lambda)}}{2(w-\lambda)} .
$$

Note that the singularity at $\lambda=w$ is removable by assigning $\rho(\lambda=w)=1-\mu / w-2 r /(w \mu)+\mu$.

At this level the adaptive SIS network model is well understood. In this paper we ask how critical transitions in adaptive network dynamics are reflected in the network topology of the underlying network(s). The practical motivation behind this question is whether it is possible to use the monitoring of the networks to infer the closeness to the critical point of adaptive systems. We are interested to what extent earlywarning signals can be derived from eventual rearrangements of network structures close to the critical transition $\lambda_{c}$. In particular we ask, whether the networks' structural changes follow certain scaling laws and if those can be used for predicting the upcoming transition. We take a first step in this direction by studying the adaptive network model proposed in Ref. [4]. Our main results are as follows:

(R1) For several network-related quantities, SI link densities, triplet densities, clustering, assortativity, and the eigenvalue gap, there exists a specific crossover of two scaling laws near criticality. As a consequence, these quantities show local extrema close to the persistence threshold. This effect can be explained within the PA framework. These extrema might indeed serve as potential candidates for network-based earlywarning signs. The eigenvalue gap might be an especially practical measure. 

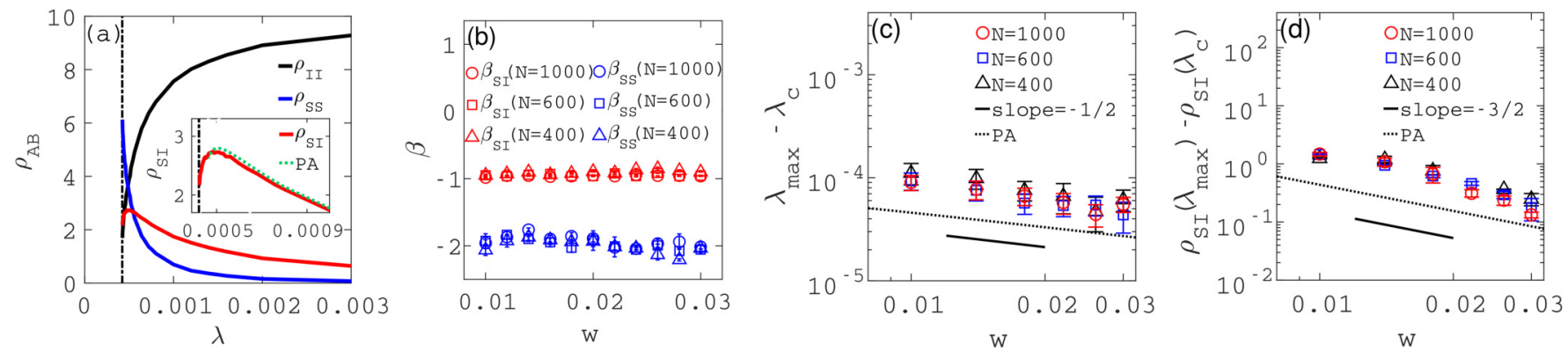

FIG. 2. (a) Critical curves of link densities are shown for numerical simulations (dashed) and in the PA (dotted). SI links (red), SS links (blue), and II links (black). $r=0.002, w=0.01,\langle k\rangle=20$, and $N=400$. The inset shows the position of the peak more clearly. (b) Exponents of the tails are fitted to $\sim \lambda^{\beta}$ for SI links (red), and SS links (blue) as a function of rewiring rates $w$ and system sizes. (c) Location and (d) size of the maxima in the SI link density as a function of $w$ and system sizes, and for reference, also their values in the PA.

(R2) Some network-related quantities, such as the degree, the effective branching ratio, and the harmonic mean distance, behave as if there existed a critical point that has a singularity located at the origin $\lambda_{c}^{\text {network }} \sim 0$. Their critical curves end abruptly at the threshold $\lambda_{c}$, so that their role as early-warning signs is limited.

(R3) Fluctuations and correlations increase for topological measures near $\lambda_{c}$, which might be an additional signal, when approaching the tipping point.

In summary, we show that topological changes in adaptive networks close to the critical point carry potential information to improve predictability of critical transitions through earlywarning signs.

\section{THE CRITICAL NETWORK}

We present the results of a numerical study of network properties near the persistence threshold. We employ the Gillespie algorithm for the simulation, which samples the stochastic process in an unbiased way [23]. For infection rates close to the threshold, we use the quasistationary method, which was used in Ref. [24], and applied to epidemic networks $[25,26]$. In this paper we focus on link densities of various link types, triplet densities, the effective branching ratio, the clustering coefficient, degree distribution, degree assortativity, compactness, and, finally, spectral properties of the adjacency matrix.

\section{A. Link densities}

The densities of SS, SI, and II links reveal a detailed picture of the mechanisms that are at work near the critical persistence threshold. In Fig. 2(a) we show the average per-node densities for SS, SI, and II links in the endemic stationary state for a range of infection rates near the persistence threshold. SS and SI link densities approach 0 asymptotically for large infection rates because rewiring cannot keep up with the infections. Hence II links dominate that regime.

Close to the persistence threshold the density of SS links (II links) increases (decrease). For the SI links, however, there is a distinctive maximum that deserves attention. One can express this observation in terms of the derivatives with respect to the infection rate. Using Eq. (2), we have $\rho_{\mathrm{SI}}^{\prime}=-\rho_{\mathrm{SS}}^{\prime}-\rho_{\mathrm{II}}^{\prime}$, where $\rho_{\mathrm{AB}}^{\prime}$ denotes the rate of change of the $\mathrm{AB}$ link density.
Thus for infection rates near the threshold the SS link density must decrease faster than II links increase; for slightly higher infection rates the roles interchange. So we conclude that SS and II links scale differently near the threshold, as can be seen in Fig. 2(b). The tail of $\rho_{\mathrm{SS}}$ scales roughly as $\lambda^{-2}$, and the tails of $\rho_{\mathrm{SI}}$ and, by link conservation, $\rho_{\mathrm{II}}$ scale as $\lambda^{-1}$. The exponent for $\rho_{\text {SI }}$ is systematically slightly overestimated, because the square-root behavior interferes slightly. This behavior is robust with respect to system size.

Using the PA in Eq. (1) we get the following estimate:

$$
\rho_{\mathrm{SI}}=\frac{r \mu}{2(w-\lambda)}\left[\sqrt{1-\frac{4 r(w-\lambda)}{\lambda^{2} \mu^{2}}}-1\right]+\frac{r}{\lambda} .
$$

Note that the singularity at $\lambda=w$ is again removable. The functional form close to the critical point is given by

$$
\rho_{\mathrm{SI}}=\frac{r}{\lambda_{c}}+\frac{\left(\lambda_{c}-2\right) r \mu \sqrt{\frac{\lambda_{c}}{2}+\frac{r}{\mu^{2}}}}{2 \lambda_{c}\left(w-\lambda_{c}\right)} \sqrt{\Delta \lambda}+\mathcal{O}(\Delta \lambda),
$$

where $\Delta \lambda=\lambda-\lambda_{c}$. Obviously, the density of SI links follows a square-root behavior near the critical point with a positive slope, as expected from the universal behavior of the fold bifurcation [27] that is present at this point [4]. For larger infection rates, $\lambda \gg \lambda_{c}$ the PA predicts a decay that is dominated by $\lambda^{-1}$. Therefore a maximum must occur in between. There are two (critical) exponents of the SI link densities. In the vicinity to the threshold we expect a squareroot behavior $\rho_{\mathrm{SI}} \sim \Delta \lambda^{\frac{1}{2}}$, and for larger $\lambda$ we get a power law decay with an exponent $\rho_{\text {SI }} \sim(\lambda-0)^{-1}$. Effectively, we can write the result obtained in Eq. (6) in the functional form,

$$
f(\lambda)=\alpha \lambda^{-\frac{3}{2}}(\Delta \lambda)^{\frac{1}{2}}+\beta \lambda^{-1}+f_{0} .
$$

The equation has three regimes. For $\Delta \lambda$ much smaller than $\lambda_{c}$, $f(\lambda) \approx \gamma(\Delta \lambda)^{1 / 2}+\delta$, with $\gamma=\alpha \lambda_{c}^{-3 / 2}$ and $\delta=\beta / \lambda_{c}+f_{0}$. When we identify $\rho_{\text {SI }}$ with $f$ we can solve for $\alpha, \beta$, and $f_{0}$ using Eq. (7). For $\lambda \gg \lambda_{c}$ it behaves as $f(\lambda) \approx \zeta \lambda^{-1}-$ $\xi \lambda^{-2}+f_{0}$ with $\zeta=\alpha+\beta$ and $\xi=\alpha \lambda_{c} / 2$, which can be checked by a Taylor expansion of Eq. (8). When we identify $\rho_{\mathrm{SI}}$ with $f$, we obtain $\zeta=r$ and $\xi=r^{2} / \mu$, again with an expansion of Eq. (6) for large $\lambda$. One can then solve for $\alpha$ and $\beta$. The intermediate regime contains the maximum. In Figs. 2(c) and 2(d) we investigate the location and height of the maximum of $\rho_{\text {SI }}$ with respect to the rewiring rate $w$. Both 

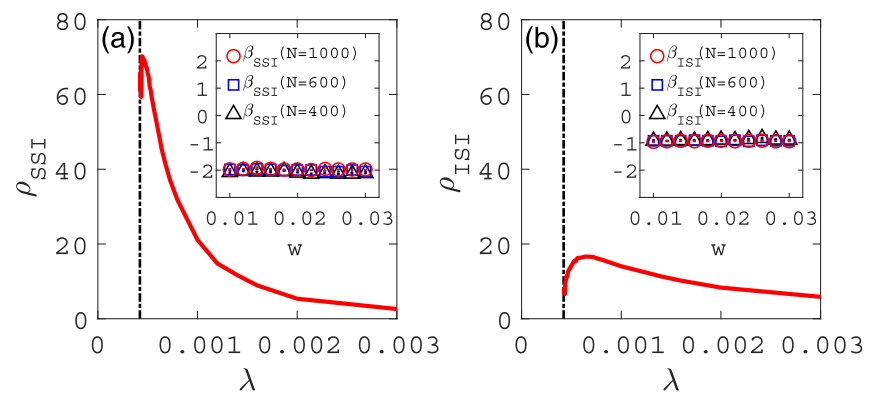

FIG. 3. (a) Density of SSI triplets. The inset shows the critical exponents of the tail for a range of rewiring rates and system sizes. It is about -2 . (b) ISI triplet density. The inset shows a critical exponent of -1 irrespective of $w$ and $N$. Parameters as before.

the distance of the maximum from the threshold and its size seem to follow a power laws $w^{-1 / 2}$ and $w^{-3 / 2}$, respectively. This behavior is also seen in the PA which is shown for comparison. Since the PA becomes less accurate towards the threshold, it is not surprising that the PA estimates differ in absolute terms but not qualitatively (slope). The behavior is again robust with respect to system size.

\section{B. Triplet densities}

We now focus on triplets where the central node is susceptible. In particular, we study the SSI and ISI motifs that are crucial in the PA in Eq. (3). Figure 3 shows the per-node densities for the SSI and ISI triplets. As for the SI links, one can distinguish three regimes: the asymptotic regime of large infection rates, the critical regime of infection rates very close to $\lambda_{c}$, and a midrange regime, containing a maximum. The maximum of the ISI triplets is further away from the threshold than in the SI link case. This can be understood in the PA, Eq. (3). The density of ISI triplets is approximately the square of the SI link densities divided by the fraction of susceptible nodes. The square of a function will not change the position of its maximum; however, division will. Consider

$$
\left(\frac{\rho_{\mathrm{SI}}^{2}}{\rho_{\mathrm{S}}}\right)^{\prime}=\frac{\left(\rho_{\mathrm{SI}}^{2}\right)^{\prime}}{\rho_{\mathrm{S}}}-\left(\frac{\rho_{\mathrm{SI}}}{\rho_{\mathrm{S}}}\right)^{2}\left(\rho_{\mathrm{S}}\right)^{\prime} .
$$

This expression is positive for the infection rate, where $\rho_{\mathrm{SI}}$ becomes maximal since the first term vanishes and the second is positive (because of the decrement of the susceptible density). Therefore the maximum of $\rho_{\text {ISI }}$ has not yet been attained at this rate. A similar analysis can be done for the SSI triplet.

\section{Effective branching ratio}

The effective branching ratio is defined as

$$
\kappa=\frac{[\mathrm{SSI}]}{[\mathrm{SI}]}=\frac{\rho_{\mathrm{SSI}}}{\rho_{\mathrm{SI}}}
$$

and quantifies the number of potential secondary infections for a given primary infection. Figure 4(a) shows $\kappa$ in log-log scale. The effective branching ratio does not have a maximum but follows a power law with an exponent $\alpha \approx-1$. The power law is clearly of the form, $\kappa \propto\left(\lambda-\lambda_{c}^{\mathrm{ebr}}\right)^{-\alpha}$, where $\lambda_{c}^{\mathrm{ebr}} \approx 0$. The critical transition at $\lambda_{c}$ is not detected by the effective branching ratio.
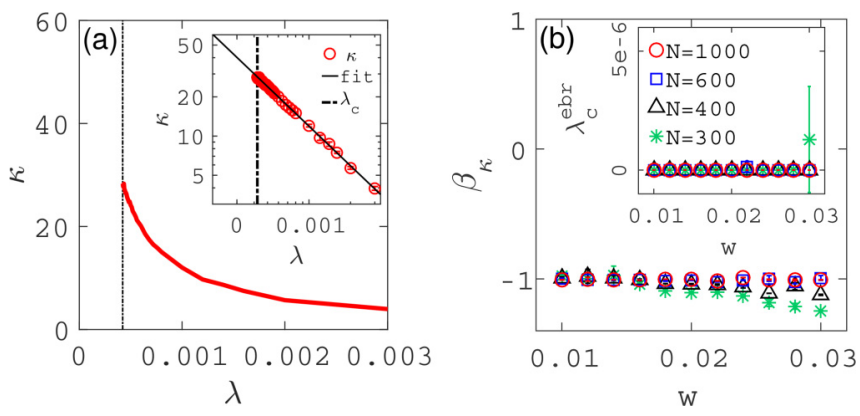

FIG. 4. (a) Effective branching ratio $\kappa=\mathrm{SSI} / \mathrm{SI}$ (red). The inset is a $\log -\log$ plot with a fitted slope, $\beta_{\kappa}=-1.01$. Parameters as before. (b) Exponent $\beta_{\kappa}$ for a range of rewiring rates and system sizes. The inset shows the fitted threshold $\lambda_{c}^{\text {ebr }}$.

We measure the exponent $\beta_{\kappa}$ for various rewiring rates $w$ and system sizes $N$ in Fig. 4(b). For finite system sizes, we find that $\beta_{\kappa}$ decreases roughly linearly with the rewiring rate. With larger system size this dependence becomes weaker. Hence, we infer that the density of SSI triplets [Fig. 3(a)] is just the product of the SI link density and a power law with exponent -1 . For secondary infections we conclude that the risk is highest just at the threshold, even though the risk of an initial infection-indicated by the SI link density-is not maximal at the critical point.

\section{Clustering coefficient}

The clustering coefficient $C$ measures the number of closed triangles with respect to the total number of triangles in the entire network. It is given in terms of the adjacency matrix $A$ of the network by

$$
C=\frac{\operatorname{Tr} A^{3}}{\sum_{i j}\left(A^{2}\right)_{i j}-\operatorname{Tr}\left(A^{2}\right)} .
$$

Figure 5(a) shows the clustering coefficient near $\lambda_{c}$. The qualitative behavior is again similar to the SI link density or the SSI and ISI triplet densities. Since there are almost no
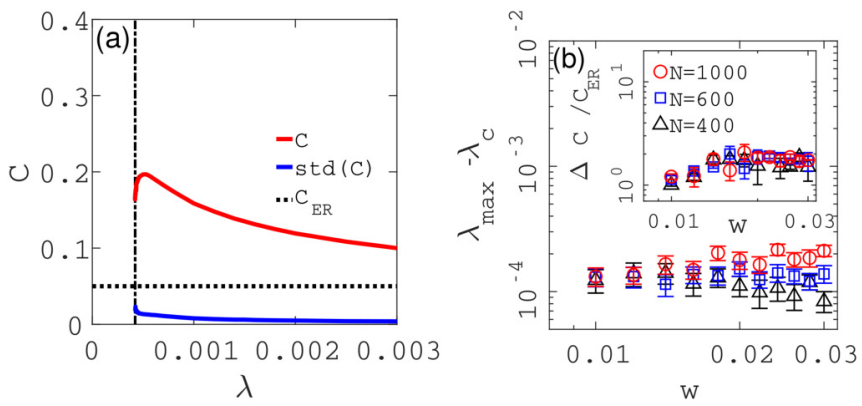

FIG. 5. (a) Clustering coefficient (red), its standard deviation around the equilibrium state (blue), and the clustering coefficient of the Erdös Rényi graph (dotted) for $N=400,\langle k\rangle=20, r=$ 0.002 , and $w=0.01$. (b) Distance and relative size (inset) of the local maximum for various $w$ and $N$. The infection rate at which the critical curve becomes maximal is $\lambda_{\max }$. Since the clustering coefficient vanishes as $N \rightarrow \infty$ at constant link density, we rescale by $C_{\mathrm{ER}}=\langle k\rangle / N$. Parameters are otherwise the same. 
susceptible nodes in the regime of large infection rates, the stationary network behaves like an Erdös Rényi graph, whose clustering coefficient is given by $C_{\mathrm{ER}}=\langle k\rangle / N$, where $\langle k\rangle=$ $1 / N \sum_{i} k_{i}=2 L / N$ and $L$ is the total number of links. $C_{\mathrm{ER}}$ is the limiting value for large infection rates (dotted horizontal line). Rewiring creates and destroys triangles. The clustering coefficient depends on the average net effect. The appearance of the maximum can be explained by this net effect in the three regimes. For high infection rates, a rewiring event has a much higher chance of closing an open triangle rather than destroying one, due to the high connectivity of the susceptible graph. However, the number of rewireable links is very low, which results in an asymptotically vanishing net effect. For infection rates very close to $\lambda_{c}$, there are many more susceptible nodes, which are only slightly more connected than the infected nodes. So the chance for creating a closed triangle is only a little bit larger than the chance to destroy one. The average net effect is nevertheless present. The largest effect occurs when the connectivity of the susceptibles is high and their abundance not too low.

Fitting a power law to the tail of $C$ is sensitive to the interval choice and whether the ER limiting value is enforced or not. Parameter values of the fitted exponent vary depending on these choices. Since the clustering coefficient is a nonlinear function of graph motifs, it is likely that multiple power laws of the respective motifs interfere, which leads to the aforementioned sensitivity.

We denote by $\lambda_{\max }$ the infection rate at which the critical curve becomes maximal. In Fig. 5(b) we show the distance of $\lambda_{\max }$ from the threshold $\lambda_{c}$ as well as the size of the maximum $\Delta C=C\left(\lambda_{\max }\right)-C\left(\lambda_{c}\right)$ as a function of the rewiring rate. The size of the maximum does not decline as a function of $w$ but levels out. However, $\Delta C \propto N^{-1}$ decreases with $N$ in the same way as the Erdös-Rényi value $C_{\mathrm{ER}}=\langle k\rangle / N$, which can be seen from the inset of Fig. 5(b). This $N^{-1}$ dependence is not surprising, because the clustering coefficient itself vanishes at constant link density as $N \rightarrow \infty$, unless there are strong preferences for local rewiring. The distance $\lambda_{\max }-\lambda_{c}$ becomes slightly larger for larger $N$. This is due to the dependence of the maximum location on the abundance of the susceptible nodes: The susceptible contribution to the clustering coefficient decreases with the absolute abundance of susceptible nodes, again due to the size dependence. Thus, for higher $N$, it requires higher infection rates to reduce this number of the susceptible nodes to a level comparable to the case of lower $N$. This effect is enhanced the more rewiring events occur, i.e., the higher $w$ becomes. In summary, the maximum of the clustering coefficient is a possible robust warning sign for the upcoming persistence threshold.

\section{E. Degree distribution}

The degree distribution $p_{k}$ is the fraction of nodes in the network with degree $k$. The average degree is $\langle k\rangle=$ $1 / N \sum_{i} k_{i}=2 L / N$. Note that $\langle k\rangle$ is constant due to the conservation of links during rewiring. The $n$th raw moment is given by

$$
\left\langle k^{n}\right\rangle=\frac{1}{N} \sum_{i} k_{i}^{n}=\rho\left\langle k^{n}\right\rangle_{\mathrm{I}}+\rho_{\mathrm{S}}\left\langle k^{n}\right\rangle_{\mathrm{S}},
$$
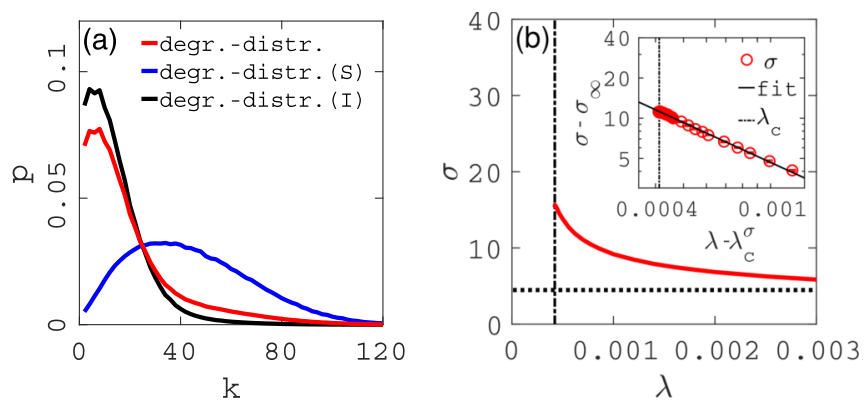

FIG. 6. (a) Degree distribution of the entire graph (red), the susceptible nodes (blue), and the infected nodes (black) for $N=1000$, $\langle k\rangle=20, r=0.002, \lambda=0.001$, and $w=0.02$. The distributions are close to Poisson distributions as one would expect from ER networks. (b) Standard deviation of the stationary degree distribution. The inset shows a power-law fit. The exponent is roughly $\beta_{\sigma} \approx-0.46$ and $\lambda_{c}^{\sigma} \approx 0.00022$ for $N=400,\langle k\rangle=20, r=0.002, w=0.01$.

where $\left\langle k^{n}\right\rangle_{\mathrm{I}(\mathrm{S})}$ are the raw moments of the degree distribution of infected (susceptible) nodes. The degree distribution in the endemic state has been studied for instance in Refs. [4,28,29]. The degrees of the infected and the susceptible nodes both follow distributions that are close to Poisson [28] (an indication for ER random graphs), however, with different mean values. The behavior in the vicinity of the phase transition at $\lambda_{c}$ has not been studied before.

Figure 6(a) shows the stationary degree distribution for a set of parameters close to the transition. The overall distribution is a superposition of the susceptible and the infected contribution. The respective Poisson-like distributions are seen. In Fig. 6(b) we show the critical curve of the standard deviation $\sigma$ at equilibrium. We observe a rise of the standard deviation close to the critical point and the absence of a local maximum. In Ref. [28] it was shown that $\operatorname{Var}>\operatorname{Var}_{\mathrm{ER}}=\langle k\rangle$ if and only if $w>\lambda$. In Fig. 6(b) the equilibrium standard deviation can be seen to be bounded from below for $w>\lambda$ by $\sigma_{\mathrm{ER}}=\sqrt{\operatorname{Var}_{\mathrm{ER}}}$, which is indicated by the dotted horizontal line.

The inset in Fig. 6(b) shows a power-law fit. The curve is well described by a power law close to the transition; however, its critical point is not $a t$ the transition. Like for the effective branching ratio (Fig. 4) the true critical point is not sensed by $\sigma$. The fitted critical points $\lambda_{c}^{\sigma}$ for various parameters and choices of intervals all share the feature that they are far away from $\lambda_{c}$ and close or equal to zero.

We conclude that the broadening of the degree distribution captures the approach towards the critical point, but the true location of the critical point $\lambda_{c}$ cannot be seen from the scaling behavior of $\sigma$.

\section{F. Degree assortativity}

Assortativity measures the correlations between the degrees of adjacent nodes. In terms of the adjacency matrix $A$ and the degree vector $k_{i}=\sum_{j} A_{i j}$ it is

$$
\mathcal{A}=\frac{\sum_{i j}\left(A_{i j}-\frac{k_{i} k_{j}}{N\langle k\rangle}\right) k_{i} k_{j}}{\sum_{i j}\left(k_{i} \delta_{i j}-\frac{k_{i} k_{j}}{N\langle k\rangle}\right) k_{i} k_{j}} .
$$



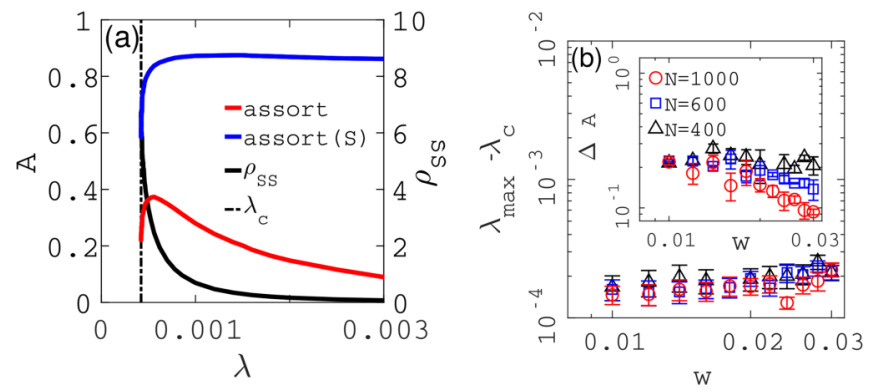

FIG. 7. (a) Assortativity coefficient for the entire graph (red) and for the susceptible graph (blue). For reference we also show the density of SS links (black), which approximates the size of the susceptible graph. $N=400,\langle k\rangle=20, r=0.002$, and $w=0.01$. (b) The distance $\lambda_{\max }$ and size $\Delta A$ (inset) of the local maxima shown for various $w$ and $N$.

It takes values between -1 and 1 . For $\mathcal{A}=0$ the network has no degree correlations, for $\mathcal{A}=1$ it is maximally degree correlated, and for $\mathcal{A}=-1$ it is maximally anticorrelated.

Figure 7(a) shows the critical curve for the assortativity. As for the SI link density and the clustering coefficient, the degree assortativity exhibits a maximum. For large infection rates the network becomes nonassortative, which is the expected Erdös-Rényi limit. At medium-range infection rates the maximum occurs. Towards the threshold, the assortativity decreases again.

It is instructive to decompose the assortativity coefficient into its constituent parts. We denote by $\left\langle k k^{\prime}\right\rangle_{\mathrm{AB}}$ the expected product of the degrees $k$ and $k^{\prime}$ along links of type $\mathrm{AB}$. In this notation the coefficient is

$$
\frac{2 \rho_{\mathrm{SS}}\left\langle k k^{\prime}\right\rangle_{\mathrm{SS}}+2 \rho_{\mathrm{SI}}\left\langle k k^{\prime}\right\rangle_{\mathrm{SI}}+2 \rho_{\mathrm{II}}\left\langle k k^{\prime}\right\rangle_{\mathrm{II}}-\frac{1}{\langle k\rangle}\left\langle k^{2}\right\rangle^{2}}{\left\langle k^{3}\right\rangle-\frac{1}{\langle k\rangle}\left\langle k^{2}\right\rangle^{2}} .
$$

The important contribution to the overall assortativity results from the first three terms in the numerator. The assortativity of the susceptible subgraph rises to high values above 0.8 , as can be seen in Fig. 7(a). This means that the most important contribution of the three terms comes from the susceptible subgraph. There is, however, a trade-off between the abundance of SS links and the expected degree correlation $\left\langle k k^{\prime}\right\rangle_{\mathrm{Ss}}$ : While the latter is increasing, the former decreases [Fig. 7(a)]. The degree correlations, however, increase faster than the SS link density decreases, thus giving rise to the maximum.

The interference of at least two scaling laws brings about the maximum. Its location is studied in Fig. 7(b). With respect to the distance of the maximum from the threshold $\lambda_{\max }-\lambda_{c}$ one observes a slight increase in distance towards higher rewiring rates, while the dependence on system size is not present or negligible. The height of the maximum $\Delta \mathcal{A}=$ $\mathcal{A}\left(\lambda_{\max }\right)-\mathcal{A}\left(\lambda_{c}\right)$ [inset of Fig. 7(b)], on the other hand, has a strong size dependence. It decreases with increasing $w$ but does so at a higher rate as the system becomes larger. The decrease might follow a power law.

The degree assortativity is a purely global quantity that is not easy to measure locally. It does, however, bear potential as an early-warning sign because the distance to the threshold is sizable and not strongly dependent on the rewiring rate or system size.

\section{G. Harmonic mean distance}

The most natural way of measuring distances on a graph is in terms of the geodesic distance. For two nodes $i$ and $j \neq i$ the geodesic distance $d_{i j}$ is the length of the shortest path between them, and is infinite if $i$ and $j$ are not connected by any path. The "farness" of a node $i$ is given by $1 /(N-1) \sum_{j \neq i} d(i, j)$ and the "closeness" by its reciprocal. For graphs with multiple connected components the farness is infinite and the closeness vanishes. To remedy this deficiency one may look at the harmonic geodesic distance $1 / d_{i j}$. The harmonic mean geodesic distance is then given by

$$
\mathrm{HMD}=\frac{1}{\left\langle\frac{1}{d}\right\rangle}=\frac{N(N-1)}{\sum_{i, j \neq i} \frac{1}{d_{i j}}} .
$$

It is 1 for a complete graph, infinite for a set of points without links, and finite otherwise.

Figure 8(a) shows the HMD. For infection rates close to the critical point, the HMD rises-the network becomes less compact. A possible explanation is that a large number of paths in the network lead through the susceptible subgraph, especially for infected nodes. This is supported by the high branching ratio $\kappa=[\mathrm{SSI}] /[\mathrm{SI}]$ close to the critical point. Therefore, the overall distances become larger in the vicinity to the critical threshold. For large infection rates the equilibrium distances approach the ensemble average of the Erdös-Rényi harmonic mean distance $\mathrm{HMD}_{\mathrm{ER}} \sim \log (\mathrm{N})$, as seen in Fig. 8(c). The susceptible subgraph itself is at its densest near the threshold and its HMD in comparison to that of an ER graph of the same size grows linearly [Fig. 8(d)]. An explanation is that the number of SS links falls by a factor of $\lambda^{-1}$ faster than the number of susceptible nodes $S=N(1-\rho)$. So the average distances of the susceptible subgraph scale by the reciprocal factor with respect to a baseline ER graph of size $S$.

Like for the effective branching ratio $\kappa$ and for the standard deviation $\sigma$ (Secs. IIC and IIE), the HMD does not sense the actual critical point $\lambda_{c}$. A fit to a power law reveals that the exponent is close to -1 for a range of rewiring rates and system sizes [Fig. 8(b)]. The fitted critical point $\lambda_{c}^{\mathrm{HMD}}$ is both bigger than 0 and strictly smaller than $\lambda_{c}$.

\section{H. Spectral properties}

The network Laplacian is defined in terms of the adjacency matrix $A$ by $L=D-A$, with $D_{i j}=\delta_{i j} \sum_{k} A_{j k}$ The largest eigenvalue of the adjacency matrix, $\lambda_{1}$, is sometimes referred to as the "capacity" of the graph. It carries information about the connectivity and the number of paths in the network. It is bounded from below by the average degree $\langle k\rangle$ and from above by the maximal degree. We denote the gap between the first two eigenvalues by $g=\lambda_{1}-\lambda_{2}$. In the context of Markov chains it measures the speed of convergence in $\ell^{p}$ to the stationary distribution under the condition of irreducibility and aperiodicity, see Refs. [30-32] for more details.

Figure 9(a) shows the four largest eigenvalues of the adjacency matrix. They all reach a maximum and then decrease towards their asymptotic values. Note, however, that the largest eigenvalue attains its maximum much closer to the threshold than the others. In Figure 9(b) we see that the eigenvalue gap is large when close to the threshold, drops to a local 

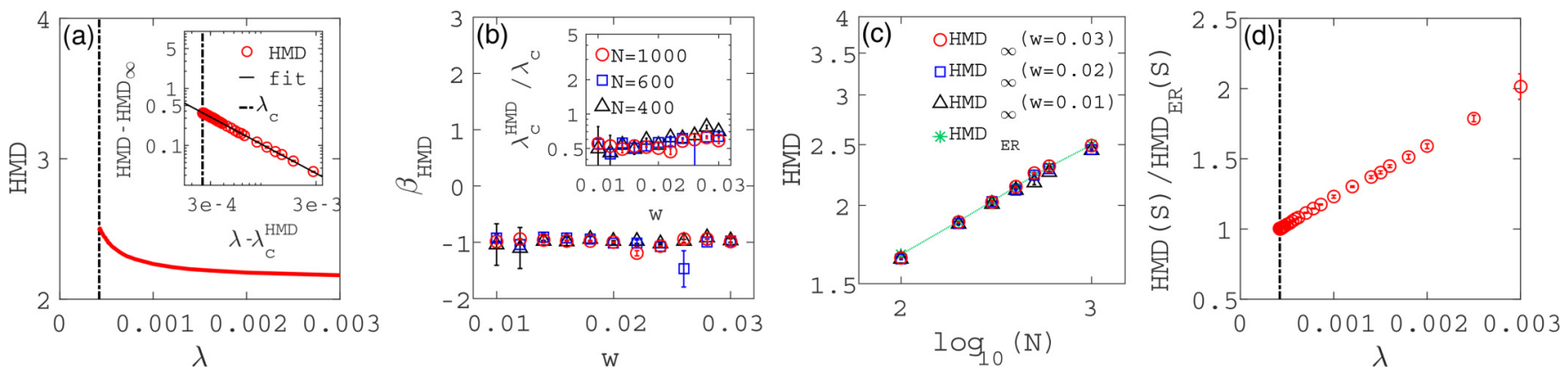

FIG. 8. (a) Harmonic mean distance HMD of the entire graph and a power-law fit on a log-log plot (inset). (b) Power-law exponent $\beta_{c}^{\text {HMD }}$ fitted to the tail and the critical point $\lambda_{c}^{\mathrm{HMD}}$ as a fraction of $\lambda_{c}$ (inset). (c) Fitted asymptotic value $\mathrm{HMD}_{\infty}$ as a function of system size $N$, with the numerical values of the Erdös-Rényi ensemble average $\operatorname{HMD}_{\mathrm{ER}}(N)$ for a reference. (d) Harmonic mean distance of the susceptible subgraph $\operatorname{HMD}(\mathrm{S})$, measured with respect to the ER value for a graph of that size $S=N(1-\rho) . N=400,\langle k\rangle=20, r=0.002$, and $w=0.01$

minimum, and then relaxes back to its asymptotic value. The extremum is around one order of magnitude further away from the threshold than the extrema of the SI link density (Fig. 2), the clustering coefficient (Fig. 5), or the degree assortativity (Fig. 7) along a comparable range of rewiring rates. The larger the gap the more difficult it is to dissect the graph [32] and the faster infections would spread. The distance of the local minimum scales linear with the rewiring rate, as can be seen from the inset of Fig. 9(b). Therefore it becomes a very reliable indicator of the transition and even more so, for higher rewiring rates.

Another important spectral characteristic of the network is the distribution of eigenvalues. It is known [33] that the empirical eigenvalue distribution of the adjacency matrix converges to the Wigner semicircle law for ER graphs. The Laplacian, however, converges to the convolution of a Gaussian with the semicircle distribution [34], after appropriate normalisation. Figure 9 shows the empirical distribution of both the adjacency matrix [Fig. 9(c)] and the graph Laplacian [Fig. 9(d)]. Far from the threshold, the eigenvalue distribution of the adjacency matrix approaches the semicircle around the origin, as expected. Close to the critical threshold the distribution changes drastically. It remains symmetric around the origin but develops a narrow peak producing a cusp at the center. This behavior is known from eigenvalue distributions of several scale-free networks [32]. For the empirical eigenvalue distribution of the Laplacian the situation is similar: An ER limit exists for high infection. Drastic changes occur near the critical threshold.

\section{DISCUSSION-USABILITY OF NETWORK MEASURES AS EARLY-WARNING SIGNS}

In summary, we formulated the general question of the feasibility of finding network-based precursor signals in adaptive network dynamics in the context of a specific epidemic model, the coevolving SIS model. We find that several network measures indicate no sensitivity whatsoever for the critical transition. These are the effective branching ratio, the degree distribution, and the harmonic mean distance. As a function of the infection rate, these measures show scaling laws, often characterized by an exponent of -1 , with the singularity located at zero or close to it but not at $\lambda_{c}$. It means that these measures behave as if the transition was at $\lambda_{c}^{\text {ebr/degree } / \mathrm{HMD}} \approx 0, \lambda_{c}^{\mathrm{ebr} / \text { degree } / \mathrm{HMD}} \ll \lambda_{c}$ rather than at $\lambda_{c}$. This has severe consequences for their use as an early warning sign, because the fold bifurcation point is suddenly reached without any warning. These measures can in no way anticipate the true position of the critical point $\lambda_{c}$.

We have shown, however, that a number of other network measures do carry potential for being used as early-warning signs. They are able to detect the critical transition at $\lambda_{c}$,
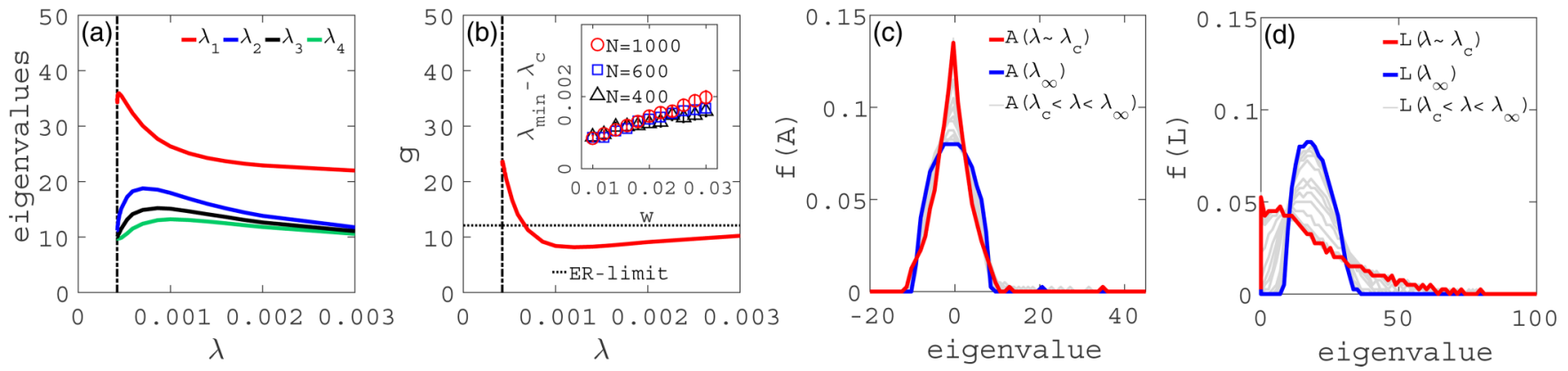

FIG. 9. (a) The first four eigenvalues. (b) The eigenvalue gap and the distances from the threshold to the minimum for a range of rewiring rates and system sizes (inset). (c) Distribution of eigenvalues of the adjacency matrix, and (d) the network Laplacian. Blue curves correspond to high values of the infection rate, $\lambda=0.03$, and show Wigner's semicircle. Red lines correspond to the distributions for infection rates close to the critical threshold, $\lambda_{c}=0.00043$. Gray lines show distributions for intermediate infection rates. $N=400,\langle k\rangle=20, r=0.002$, and $w=0.01$. 
when approached from above $\left(\lambda>\lambda_{c}\right)$. In particular, these measures, which include the SI link densities, triplet densities, clustering, and assortativity, show a crossover of two scaling laws that are of a functional form as in Eq. (8). The first scaling law shows an increase of the respective measure as $\left(\lambda-\lambda_{c}\right)^{1 / 2}$, slightly above the transition $\left(\lambda>\lambda_{c}\right)$. The other is an asymptotic scaling law $\left(\lambda \gg \lambda_{c}\right)$, which is characterized by negative integer exponents. Between these two scaling regimes a local maximum exists, which is indeed visible in the corresponding network measures and which is in line with the pair approximation in the case of SI link densities. The location of the maxima occur slightly above the critical point, $\lambda_{\max }>\lambda_{c}$.

Both the double scaling and the maximum is also seen in the maximum eigenvalue of the adjacency matrix, when plotted against $\lambda$. The eigenvalue gap shows a very clear minimum, well before the critical transition. In practical terms this means that, when approaching the critical transition point, an increase of the eigenvalue gap signals the immediate vicinity of the transition. Given that a method to detect the eigenvalue gap is available, such as the equilibration rate of a Markov process on the network, or a sufficiently robust eigenvalue estimate from data, the eigenvalue gap is a very clear and practical early-warning sign.

Regarding advantages and disadvantages of the discussed precursor signals, we draw the following conclusion: Compared to the other quantities, the SI link density is a shortrange precursor. Its signal depends strongly on the rewiring rate, making it unsuitable for high rewiring rates. The SI link density can, however, be measured quite well locally. The clustering coefficient produces a signal that does not strongly dependent on the rewiring rate. Ignorance about the rewiring rate is not a big problem for this signal. One can measure the coefficient reasonably well from local connectivity information. The assortativity coefficient suffers from its global character, making it a hard to measure locally. Nevertheless, it performs well for higher rewiring rates and smaller system sizes. The eigenvalue gap outperforms all measures in its ability to detect the signal very early; however, it hinges on the measurability of eigenvalues, which can be hard in practice.

We tested the effects of all parameters in the coevolving SIS model and found that our results are relatively robust. The dependence on rewiring rate and system size has been has been investigated especially carefully. The recovery rate sets the time scale and can therefore can be fixed arbitrarily; we took the choice used in Ref. [4]. The connectivity determines the location of the threshold. The homogeneous pair approximation becomes unreliable for very low values of $\langle k\rangle$ [29].
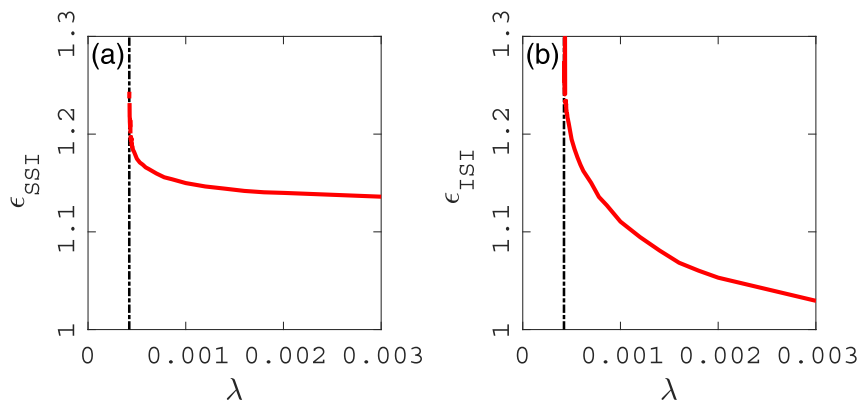

FIG. 10. Ratio of the exact triplet densities and their pair approximation is plotted at stationarity (a) for $\varepsilon_{\mathrm{SSI}}$ and (b) $\varepsilon_{\mathrm{ISI}}$. $N=400$, $\langle k\rangle=20, r=0.002$, and $w=0.01$.

We conclude by mentioning that the network information that is neglected in the classical coarse-graining approach does contain a layer of structural information that can indeed detect the critical transition point. The next steps to take would be to actually test the performance of the different network measures as precursor signals in agent-based simulations, where infection rates are exogenously varied slowly. An observer can monitor the networks, the infection, and the rewiring rates but would not know anything about the location of the critical point. It would be interesting to see to what extent such an observer could predict the collapse of the system several time steps in advance.

\section{ACKNOWLEDGMENT}

Supported by the Austrian Science Foundation FWF under Project No. P29252.

\section{APPENDIX: QUALITY OF THE PAIR APPROXIMATION}

In simulations the quality of the homogeneous pair approximation can be measured directly through the ratio of the exact triplet density over the approximate triplet densities,

$$
\varepsilon_{\mathrm{SSI}}=\frac{\rho_{\mathrm{SSI}}}{2 \rho_{\mathrm{SI}} \rho_{\mathrm{SS}} / \rho_{\mathrm{S}}}, \quad \varepsilon_{\mathrm{ISI}}=\frac{\rho_{\mathrm{ISI}}}{\rho_{\mathrm{SI}} \rho_{\mathrm{SI}} / \rho_{\mathrm{S}}} .
$$

For ratios above 1 the approximation underestimates the true triplet density. Figure 10 indicates that the quasistationary density of SSI and ISI triplets is systematically underestimated, which confirms the conjecture that moment closure becomes problematic near the instability [15]. Further, it shows that correlations between different moment orders indicate proximity to the persistence threshold.
[1] T. Gross and B. Blasius, J. Roy. Soc. Interface 5, 259 (2008).

[2] T. Gross and H. Sayama, editors, Adaptive Networks: Theory, Models and Applications (Springer, Berlin, 2009).

[3] G. Szabó and G. Fath, Phys. Rep. 446, 97 (2007).

[4] T. Gross, Carlos J. Dommar D'Lima, and B. Blasius, Phys. Rev. Lett. 96, 208701 (2006).
[5] L. B. Shaw and I. B. Schwartz, Phys. Rev. E 77, 066101 (2008).

[6] J. M. Pacheco, A. Traulsen, and M. A. Nowak, Phys. Rev. Lett. 97, 258103 (2006).

[7] C. Biely, K. Dragosits, and S. Thurner, Physica D 228, 40 (2007). 
[8] M. G. Zimmermann, V. M. Eguíluz, and M. San Miguel, Phys. Rev. E 69, 065102 (2004).

[9] C. Biely, R. Hanel, and S. Thurner, Eur. Phys. J. B 67, 285 (2009).

[10] S. Bornholdt and T. Rohlf, Phys. Rev. Lett. 84, 6114 (2000).

[11] A. Levina, J. M. Herrmann, and T. Geisel, Nat. Phys. 3, 857 (2007).

[12] S. Poledna and S. Thurner, Quant. Financ. 16, 1599 (2016).

[13] S. Jain and S. Krishna, Phys. Rev. Lett. 81, 5684 (1998).

[14] J. P. Gleeson, Physical Review X 3, 021004 (2013).

[15] C. Kuehn, Moment closure-A brief review, in Control of SelfOrganizing Nonlinear Systems, edited by E. Schöll, S. Klapp, and P. Hövel (Springer, Berlin, 2016), pp. 253-271.

[16] G. Demirel, F. Vazquez, G. A. Böhme, and T. Gross, Physica D 267, 68 (2014).

[17] F. Vazquez, V. M. Eguíluz, and M. S. Miguel, Phys. Rev. Lett. 100, 108702 (2008).

[18] K. Wiesenfeld, J. Stat. Phys. 38, 1071 (1985).

[19] C. Kuehn, G. Zschaler, and T. Gross, Sci. Rep. 5, 13190 (2015).

[20] C. Kuehn, J. Nonlinear Sci. 23, 457 (2013).

[21] R. Pastor-Satorras and A. Vespignani, Phys. Rev. E 63, 066117 (2001).
[22] T. Gross and I. G. Kevrekidis, Europhys. Lett. 82, 38004 (2008).

[23] D. Gillespie, J. Comput. Phys. 22, 403 (1976).

[24] M. M. de Oliveira and R. Dickman, Phys Rev. E 71, 016129 (2005).

[25] S. C. Ferreira, C. Castellano, and R. Pastor-Satorras, Phys. Rev. E 86, 041125 (2012).

[26] S. C. Ferreira, R. S. Ferreira, and R. Pastor-Satorras, Phys. Rev. E 83, 066113 (2011).

[27] J. Guckenheimer and P. Holmes, Nonlinear Oscillations, Dynamical Systems, and Bifurcations of Vector Fields (Springer, New York, 1983).

[28] S. Wieland and A. Nunes, Analytic description of adaptive network topologies in a steady state, Phys. Rev. E 91, 060801 (2015).

[29] V. Marceau, P.-A. Noël, L. Hébert-Dufresne, A. Allard, and L. J. Dubé, Phys. Rev. E 82, 036116 (2010).

[30] D. A. Levin and Y. Peres, Markov Chains and Mixing Times (AMS, Providence, RI, 2017), Vol. 107.

[31] F. R. K. Chung, Spectral Graph Theory (AMS, Providence, RI, 1996).

[32] F. R. K. Chung and L. Lu, Complex Graphs and Networks (AMS, Providence, RI, 2006).

[33] Z. Füredi and J. Komlós, Combinatorica 1, 233 (1981).

[34] X. Ding and T. Jiang, Ann. Appl. Prob. 20, 2086 (2010). 